Vittalle - Revista de Ciências da Saúde v. 32, n. 2 (2020) 140-147

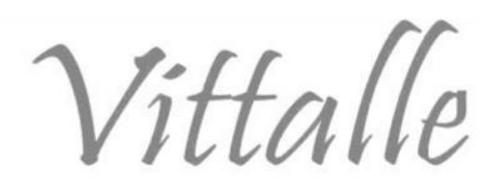

\title{
Coração como centro das emoções e da vida: um estudo com pacientes cardiopatas em hospitais gerais
}

\author{
Thalita Saramago de Souza ${ }^{a^{*}}$, Pamela Staliano ${ }^{\mathrm{b}}$
}

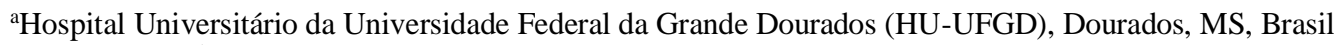

bUniversidade Federal da Grande Dourados (UFGD), Dourados, MS, Brasil

\section{Histórico do Artigo \\ Recebido em: \\ 01/12/2019}

Aceito em:

$10 / 04 / 2020$

Palavras-chave:

Psicologia;

cardiologia;

simbolismo

\begin{abstract}
RESUMO
As doenças cardiovasculares são a principal causa de morte no mundo. Levando em consideração os simbolismos atribuídos ao coração, muitas são as consequências psicológicas associadas às doenças cardíacas. Assim, considera-se de extrema importância compreender os simbolismos associados a este órgão. Portanto, este estudo teve como objetivo discutir os simbolismos que pacientes cardíacos possuem acerca do coração. Trata-se de um estudo qualitativo de cunho exploratório realizado em um hospital universitário público e em um hospital privado referência para cirurgia cardíaca, localizados no município de Dourados/MS, durante o período de setembro de 2018 a julho de 2019. Os registros pessoais foram submetidos à análise de conteúdo e respeitou os passos da técnica proposta por Bardin. Assim, foram elencadas as seguintes categorias de análise: a) Coração como centro das emoções e da vida; b) O coração e a necessidade de reparação e, c) Desintegração do corpo e do coração. Os simbolismos atribuídos ao coração por pacientes cardíacos perpassam pela compreensão desse ser um órgão ligado, intrinsecamente, à vida e às emoções, e, portanto, a manipulação deste órgão culmina na desintegração simbólica do corpo e do próprio ego, gerando diversas manifestações clínicas e psicológicas. Nesta perspectiva, ao compreender os simbolismos que pacientes cardiopatas possuem acerca do próprio coração, é possível definir, de modo mais efetivo, as intervenções psicológicas para que a hospitalização, quando necessária, e a convivência com a doença não gerem alterações emocionais/psicológicas incapacitantes.
\end{abstract}

Heart as a center of emotions and life: a study with cardiopathy patients in general hospitals

\section{ABSTRACT}

Cardiovascular diseases are the leading cause of death in the world. Taking into account the symbolisms attributed to the heart, there are many psychological consequences associated with heart disease. Thus, it is considered extremely important to understand the symbolisms associated with this organ. Therefore, this study aims to discuss the symbolisms that cardiac patients have about the heart. This is a qualitative exploratory study carried out in a public university hospital and in a private reference hospital for cardiac surgery, located in the city of Dourados / MS, from September 2018 to July 2019. Personal records were submitted to content analysis and respected the steps of the technique proposed by Bardin. Thus, the following categories of analysis were listed: a) Heart as the center of emotions and life; b) The heart and the need for repair, and c) Disintegration of the body and heart. The symbolism attributed to the heart by cardiac patients permeates the understanding of this being an organ intrinsically linked to life and emotions, and, therefore, the manipulation of this organ culminates in the symbolic disintegration of the body and the ego itself, generating several clinical and psychological manifestations. . In this perspective, by understanding the symbolisms that cardiac patients have about their own heart, it is possible to define, more effectively, psychological interventions so that hospitalization, when necessary, and living with the disease do not generate disabling emotional / psychological changes.

\section{Introdução}

As doenças cardiovasculares são a principal causa de morte no mundo, sendo as mais recorrentes a cardiopatia isquêmica e o acidente vascular cerebral, responsáveis por um total de 15,2 milhões de óbitos em 2016, resultando no crescente índice de internação e

\footnotetext{
* Autor correspondente: thali.saramago@gmail.com (Souza T.S.)
} 
cirurgia cardíaca em hospitais gerais e centros especializados, conforme a Organização Pan-Americana de Saúde (1). De acordo com o Ministério da Saúde (2), em 2020, 34\% de todos os óbitos terão como causa alguma doença cardiovascular.

Desde a década de 1940, diversos autores já descreviam traços de personalidade relacionados a determinadas doenças. Nesta perspectiva, foram classificados tipos de personalidade que são associados a processos de adoecimento. Os tipos de personalidade mais descritos na literatura são os tipos A e C. A personalidade tipo C é muito relacionada com a propensão para neoplasias, caracteriza-se por comportamentos rígidos, presença de sentimentos negativos e tentativas de dissimular sentimentos positivos (3).

À medida que a personalidade do tipo $\mathrm{C}$ é associada com as neoplasias, estudiosos especificam a personalidade tipo A como maior risco para diagnóstico de doenças coronarianas. São comuns comportamentos mais impulsivos e imediatistas, além de competitividade e tensão exacerbada (3). Nesta perspectiva, observa-se que o processo de adoecimento está intimamente ligado com aspectos psicológicos e emocionais, além de aspectos orgânicos.

É sabido que, fisiologicamente, todas as funções físicas e modulação de comportamentos e sentimentos estão localizadas no cérebro, no entanto, o coração é o órgão simbolizado como detentor dos sentimentos. Levando em consideração tais significados e simbolismos atribuídos ao coração, muitas são as consequências psicológicas associadas às doenças cardíacas (4). Assim, considera-se de extrema importância compreender os simbolismos associados a este órgão.

Diante deste cenário surgiu o interesse deste estudo, uma vez que a compreensão que os pacientes possuem sobre o coração influencia o tratamento e prognóstico, bem como, a qualidade de vida deste sujeito. Neste sentido, utiliza-se a perspectiva psicanalítica como referencial teórico para compreender os simbolismos associados ao coração, bem como, as intervenções do psicólogo diante deste acontecimento e as discussões realizadas neste estudo. A definição desta abordagem teórica deu-se por esta se apresentar como abordagem utilizada pelas autoras para a compreensão do sujeito e seu funcionamento psíquico.

Este trabalho adota a metodologia do estudo qualitativo de cunho exploratório, cujo objetivo consiste em aproximar-se do objeto de estudo e proporcionar uma compreensão geral acerca da temática (5). Portanto, este estudo objetivou discutir os simbolismos que pacientes submetidos e/ou com indicação de cirurgia cardíaca possuem acerca do coração e sua subjetividade.

\section{Método}

Este estudo é parte integrante do Trabalho de Conclusão de Residência de um Programa de Residência Multiprofissional em Saúde, do município de Dourados/MS, apresentado como requisito obrigatório para obtenção de título de especialista em Atenção Cardiovascular. Foi submetido e aprovado pela Comissão de Avaliação de Pesquisa e Extensão do hospital em que a experiência foi vivenciada, sob o parecer $\mathrm{n}^{\mathrm{o}}$ 85.2018.

Trata-se de um estudo realizado em um hospital universitário público e um hospital privado referência para cirurgia cardíaca, localizados no município de Dourados/MS, durante o período de setembro de 2018 a julho de 2019. A escolha dos hospitais deu-se a partir da realização dos estágios de uma psicóloga residente em um Programa de Residência Multiprofissional em Saúde de um Hospital Universitário, locais préestabelecidos em cronograma específico do Programa. 
O acompanhamento psicológico foi realizado durante o período de hospitalização dos pacientes. Deu-se início a partir da solicitação do paciente, da equipe assistente, e ainda, a partir da busca ativa realizada pela psicóloga. O acompanhamento psicológico, no serviço hospitalar, tem como objetivo avaliar aspectos que influenciam o processo de adoecimento/tratamento, bem como, auxiliar o indivíduo a percorrer este processo de forma funcional e adaptativa.

Neste sentido, os atendimentos psicológicos foram realizados à beira-leito, assim como, nos corredores do hospital, salas de espera, centro cirúrgico, solários e outros espaços em que os pacientes transitavam. Os atendimentos foram realizados conforme a demanda e o tempo de internação, sendo registrados em prontuários multiprofissionais, a fim de que a comunicação e as discussões das diversas categorias profissionais que prestaram atendimento ao paciente fossem facilitadas.

Este estudo parte, então, das intervenções psicológicas realizadas com pacientes em tratamento nestas instituições no período supracitado. Atende aos seguintes critérios de elegibilidade: a) ter indicação para cirurgia cardíaca; b) mais de 12 anos de idade; c) não ser indígena. A não participação de pacientes indígenas é justificada pelo fato da dificuldade em realizar interpretações e generalizações com os pacientes não-indígenas, uma vez que essa população possui sua própria linguagem, sendo a língua materna muito utilizada pelos pacientes, sua relação com o processo de hospitalização e adoecimento, e ainda, sua produção de saúde.

Baseou-se na perspectiva de compreender a percepção e o simbolismo que os pacientes submetidos à cirurgia cardíaca possuem acerca do coração. Os dados foram coletados durante os atendimentos com os pacientes. Utilizou-se uma entrevista semiestruturada para a coleta dos dados sociodemográficos. Os dados acerca do funcionamento psíquico e da personalidade dos pacientes foram obtidos a partir dos relatos dos mesmos durante os atendimentos e da observação ativa realizada durante o processo de internação hospitalar.

Todos os dados foram registrados em arquivos pessoais da profissional, assim como as demais informações obtidas, a fim de realização das análises. Nestes registros, também foram anotadas as percepções da residente sobre os atendimentos realizados, conteúdos a serem analisados e discutidos no momento da produção deste trabalho.

Os registros pessoais foram submetidos à análise de conteúdo e respeitou os passos da técnica proposta por Bardin (6): 1) pré-análise; 2) exploração do material; 3) tratamento dos dados, inferência e interpretação. Assim, após o tratamento e interpretação dos dados obtidos, foram elencadas as seguintes categorias de análise: a) Coração como centro das emoções e da vida: nesta categoria, foram agrupados os registros dos atendimentos em que os pacientes sinalizam a simbologia do coração como centro das emoções e da vida, que vai ao encontro das percepções produzidas desde a antiguidade, quando este órgão era representado como detentor da vida; b) $O$ coração $e$ a necessidade de reparação: nesta categoria, foram apontados os registros em que os pacientes buscam retomar o controle das suas vidas, uma vez que, simbolicamente, o coração representa este controle e deverá ser manipulado pelo outro, quando há a necessidade de intervenção cirúrgica. Para isso, o conceito psicanalítico de reparação é utilizado para discutir este processo; e c) Desintegração do corpo e do coração: nesta categoria, foram apresentados os registros dos atendimentos em que os pacientes simbolizam o coração como representação do próprio ego, havendo a dificuldade de conceber a desintegração deste órgão.

\section{Resultados e discussão}


Durante o período da atuação da psicóloga nas instituições, trinta e cinco pacientes cardiopatas foram atendidos, todos provenientes do município de Dourados/MS e região. Estes pacientes possuíam diversas doenças cardíacas, dentre elas: as Valvopatias, Aneurismas Torácicos, Doenças Arteriais Coronarianas, Insuficiência Cardíaca e Arritmia Cardíaca.

Não se verificou diferença significativa dos resultados obtidos com os pacientes internados no hospital universitário e no hospital particular, neste sentido, todos os dados foram agrupados e interpretados nas categorias de análise sem distinção acerca das instituições hospitalares.

Com relação aos dados sociodemográficos, foram atendidos pacientes com idade entre 24 e 90 anos, com a prevalência de pacientes idosos (68,57\%), do sexo masculino $(57,15 \%)$, casados $(82,85 \%)$, e sem diagnóstico prévio de psicopatologias, como depressão e transtorno de ansiedade $(71,42 \%)$. Entretanto, muitos pacientes faziam uso de psicotrópicos. Este dado pode ser explicado devido à falta de diagnóstico realizado por profissional especialista, sendo estes pacientes medicados por clínicos gerais e médicos de outras especialidades.

Os dados obtidos sobre o perfil psicológico destes pacientes mostram que houve maior presença de características da personalidade tipo A, em detrimento da personalidade tipo B. A primeira, também conhecida como personalidade coronariana, devido a sua grande relação com doenças coronarianas, é descrita como a personalidade de cunho autoritário, com prevalência de comportamentos ambiciosos, imediatistas, competitivos e hiperativos. Indivíduos com estes traços de personalidade tendem a apresentar entonação emotiva e explosiva ao falar, hiperinvestimento profissional e incapacidade de relaxamento satisfatório, ainda que reserve tempo para descanso em sua rotina (3).

O segundo tipo de personalidade (tipo B) é pouco descrito na literatura, sendo utilizado apenas como comparação com relação ao traço de personalidade tipo A. É caracterizado por comportamentos mais passivos, reflexivos, sendo observados em indivíduos com maior investimento relacional e afetivo e menor necessidade de urgência e imediatismo (7). Durante a situação de crise, estabelecida após a hospitalização e notícia da necessidade de cirurgia cardíaca, observa-se que pessoas com personalidade do tipo B aproximam-se dos familiares e rede de apoio, o que favorece o enfrentamento funcional e adaptativo, apresentando mais sentimentos de confiança, esperança e autoeficácia.

Durante os atendimentos realizados, percebeu-se a presença de sentimentos comuns, independente das doenças cardíacas, procedimento cirúrgico a ser realizado, prognóstico e traços de personalidade. Este dado se relaciona às representações simbólicas que foram construídas sobre o coração, ao longo do tempo. Neste sentido, destacam-se as principais categorias sobre os simbolismos que os pacientes expressam do coração, simbolizado, de modo geral na literatura, como órgão detentor da vida (8).

\section{Coração como Centro das Emoções e da Vida}

Desde a antiguidade, o coração é associado à ideia de força e centro das emoções. Quando, por exemplo, Aristóteles (384-322 a. C.) afirmava que o coração era a fonte das emoções e sensações, e, portanto, seria o último órgão a morrer. Em pesquisa realizada por Amui, Otto e Mestre (9) com pacientes internados em um hospital no sul do Brasil, evidenciou-se que, no campo da análise de conteúdos do inconsciente, ao realizar o movimento de olhar para o coração doente, os pacientes também se perceberam olhando para a própria psique e para a própria vida, permitindo-lhes uma transformação e (re)significação sobre a vida e o mundo. Esta (re)significação é 
percebida durante o relato de um atendimento abaixo:

Durante o atendimento psicológico com um paciente a ser submetido à cirurgia de substituição de válvula aórtica, percebi que o paciente realizava elaborações sobre produção e manutenção de sua própria saúde. Ao comparar sua cirurgia com a vida na cidade grande (trânsito caótico e violência), refletia que, com relação à cirurgia, também poderia sair de casa sem saber se voltaria, mas caso retornasse, deveria dar mais valor à sua família e adotar um estilo de vida mais saudável (Registros pessoais da residente, em fevereiro de 2019).

Os resultados da pesquisa de Amui et al. (9) vão ao encontro da discussão de Cardoso et al. (10) sobre cirurgia cardíaca. Para estes autores, o coração contém emoção e personalidade e, simbolicamente, está associado com a vida e, portanto, com o corpo. Este processo cíclico, inclusive, pode influenciar o sucesso de procedimentos cirúrgicos.

Tais percepções também são implicadas a pacientes portadores de arritmias cardíacas que necessitam do implante do Marca-passo, tratamento indicado para correção das síndromes bradicárdicas. Neste caso, as complicações psicológicas podem estender-se ao pós-operatório, pois podem instalar-se fantasias e inseguranças relacionadas à possibilidade de mau funcionamento do aparelho, significando a marca da fragilidade do próprio coração e conferindo um teor de desnaturalização da vida (11).

Para Angerami-Camon (12), quanto mais importante for o órgão a ser operado, para o paciente, maior será a ansiedade relacionada à cirurgia, portanto, ao se tratar da manipulação do "centro das emoções e da vida", o paciente encontra-se em uma situação de crise, potencializada por sentimentos negativos, como a ansiedade e o medo. Este medo não está relacionado, apenas à morte, mas também à anestesia, ao pósoperatório em UTI e às possíveis mudanças no cotidiano do paciente (13).

$\mathrm{O}$ medo da morte, sentimento muito presente nos pacientes atendidos configura-se como marco deste momento. Este sofrimento psíquico relacionado à morte foi explicado por Freud (14), quando afirma que o inconsciente

[...] desconhece tudo o que é negativo e toda e qualquer negação; nele as contradições coincidem. Por este motivo, não conhece sua própria morte [...] [afirma, também, ser] impossível imaginar nossa própria morte [...]. Por isso, a escola psicanalítica pôde aventurar-se a afirmar que no fundo ninguém crê em sua própria morte, ou, dizendo a mesma coisa de outra maneira, que no inconsciente cada um de nós está convencido de sua própria imortalidade [e onipotência] (Freud, 1915, p. 297).

\section{O Coração e a Necessidade de Reparação}

Por se tratar da manipulação de um órgão tão valorizado, a cirurgia cardíaca, quando necessária, obriga o paciente a fazer uma reavaliação da vida como um todo (3), tornando-o um espectador de suas vivências prévias, transformações, defeitos, erros e acertos.

Tal movimento pode causar angústia e sentimento de impotência, pelo fato de que o indivíduo não pode mudar o passado e também não lhe compete à cura de sua doença. Devido à perda do controle da situação, pacientes cardiopatas tendem a reviver conflitos anteriores, no intuito de reparar danos causados e recuperar, em algum nível, o controle de áreas da sua vida. Neste contexto, o conceito de reparação (15), proposto por Melanie Klein, perpassa pela necessidade do paciente de restaurar o objeto, podendo ser 
ele: sua própria saúde, relações afetivas, e ainda, conflitos psíquicos anteriores. Este movimento é retratado nos seguintes relatos:

\begin{abstract}
RELATO 1
Ao atender o paciente, recém-diagnosticado com insuficiência valvar e endocardite, com indicação cirúrgica de troca de válvula mitral, ele me questiona sobre ter problema no coração, um órgão tão delicado, mas também, um órgão sério.

No questionamento do paciente, observei que o mesmo demonstrou a sua percepção de fragilidade do corpo e do próprio ego, possui muita dificuldade em elaborar conteúdos subjetivos, mas expressa o desejo de reparar aspectos familiares e sociais (Registros pessoais da residente, em outubro de 2018).
\end{abstract}

\title{
RELATO 2
}

Durante o acompanhamento psicológico, um paciente infartado trazia muitos conflitos familiares, principalmente, relacionados aos filhos, com quem não mantinha contato há vários anos. No dia anterior à cirurgia de Revascularização do Miocárdio, apresentava-se satisfeito e menos ansioso, pois havia ligado para os filhos e reparado a separação, estabelecida há oito anos. Percebi que esta reparação foi motivada por sentimentos relacionados à morte e à necessidade de ir para o Centro Cirúrgico sem assuntos inacabados.

Minhas impressões foram de que, naquele momento, a desorganização psíquica deste paciente foi tamanha que o permitiu perpassar, inclusive, por estágios do luto estabelecido pelas diversas perdas simbólicas vivenciadas (Registros pessoais da residente, em fevereiro de 2019).

O coração, aqui simbolizado como um marco de que a vida é passageira e da impotência diante do adoecimento, guia o paciente a uma retrospectiva de sua própria vida. Neste momento, é comum a presença do sentimento de culpa por "não ter cuidado da saúde o suficiente" e, por isso, necessitar da intervenção cirúrgica. A culpa também surge diante de conflitos não resolvidos e vivências do passado. Grisa e Monteiro (16) discutem que o sentimento de culpa tem o seu desenvolvimento firmado na perda simbólica de aspectos familiares, econômicos e sociais.

\section{Desintegração do Corpo e do Coração}

Luchina (17) salienta que, simbolicamente, a mente é capaz de vivenciar a desintegração e, até mesmo, a perda dos órgãos, com exceção do coração, uma vez que, está associado com a vida. Portanto, se este para de funcionar, o indivíduo depara-se com o fim da vida física e psíquica. Neste caso, torna-se um órgão único e insubstituível; e, se por um lado, esta percepção, na maioria das vezes, não se dá de forma consciente, por outro lado, há aqueles que conseguem verbalizar este movimento por meio de metáforas, como elucidado nos registros pessoais da residente:

No atendimento com um paciente portador de aneurisma aórtico de $6,2 \mathrm{~cm}$ (considerado aneurisma com risco elevado de rompimento), ele refere que a perna, braço e outros órgãos podem ser trocados, o que não acontece com o coração, com este órgão tão complexo, tudo poderia acontecer no momento da cirurgia.

Portanto, com esta reflexão do paciente, fica evidenciada a 
dificuldade do mesmo com relação à cirurgia cardíaca e a necessidade de pensar na desintegração deste órgão tão valioso (Registros pessoais da residente, em fevereiro de 2019).

Este movimento, ainda que não consciente, gera grande ansiedade e angústia, pois a imagem que o paciente tem de si, além da forma como se coloca no mundo são abaladas ao se perceber entre a vida e a morte, diante da ruptura que se institui neste momento da sua vida (3).

Neste cenário, o psicólogo deve, a partir da escuta, de instrumentos e de estratégias do saber psicológico, compreender tais percepções para além do entendimento orgânico do sujeito, concedendo-lhe espaço para depreender sobre a subjetividade envolvida neste órgão detentor da vida e das emoções.

\section{Considerações finais}

Os simbolismos atribuídos ao coração por pacientes cardíacos perpassam pela compreensão desse ser um órgão ligado, intrinsecamente, à vida e às emoções, e, portanto, a manipulação deste órgão ocasiona na desintegração simbólica do corpo e do próprio ego. O coração de pacientes cardíacos, ainda, é simbolizado como um marco de impotência e perda de controle de suas próprias vidas, sendo necessária a regressão e retomada de aspectos anteriores na tentativa de reparação.

Tais percepções podem gerar, durante a internação hospitalar e ao decorrer da vida, manifestações clínicas e psicológicas que potencializem os sintomas clássicos da doença cardíaca, estando associado a piores desfechos clínicos e baixa qualidade de vida. Nesta perspectiva, ao compreender as percepções e simbolismos que pacientes cardiopatas possuem acerca do próprio coração, é possível definir, de modo mais efetivo, as intervenções psicológicas necessárias para que a hospitalização, quando necessária, não gerem alterações emocionais/psicológicas incapacitantes.

Além disso, compreender o simbolismo associado ao coração permite que o psicólogo auxilie o paciente a perpassar pelo processo de adoecimento de forma funcional, contribuindo para a melhora da qualidade de vida deste sujeito. Desta forma, não se pretende com este estudo esgotar as possibilidades de compreensão deste tema atual e necessário. É essencial que novas produções sejam realizadas, bem como, ferramentas de avaliação e intervenção na grande área da Psicologia da Saúde, com o intuito de fomentar esta discussão.

\section{Referências}

1. Organização Pan-Americana da Saúde. Saúde nas Américas. Resumo do panorama regional e perfil do Brasil. Washington, D.C.: OPAS, 2018. Disponível em https://www.paho.org/bra/index.php?option=com_content $\&$ view=article \&id=5638:10-principaiscausas-de-morte-no-mundo\&Itemid=0. Acessado em novembro de 2019.

2. Brasil. Ministério da Saúde. DATASUS. Departamento de Informática do SUS, 2015

3. Martins MCA. Emoção... Emoções... que implicações para a saúde e qualidade de vida? Millenium journal of Education, Technologies, and Health, 2016; 34 (13): 125-148.

4. Andreis M, Ebaid C. A intervenção do psicólogo junto a pacientes cardiopatas. Arq Bras Cardiol 1990; 55(2): 133-135.

5. Gil AC. Métodos e técnicas de pesquisa social. São Paulo: Atlas; 2008.

6. Bardin L. Análise de conteúdo. Lisboa: Edições 70; 2008.

7. Étienne A, Fontaine O, Kulbertus H. Stress e Cardiologia. Lisboa: Climepsi, 1998.

8. Santos LFR, Pereira MIF, Martins CV. As doenças do coração e as emoções: conversações entre a 
psicossomática e a psicologia analítica. Self - Revista do Instituto Junguiano de São Paulo, 2017; 2(5): 1-15.

9. Amui JM, Otto FF, Mestre M. Coração e alma: vida e outros símbolos das doenças cardíacas na realidade hospitalar. Rev. SBPH 2012; 15(1): 114-133.

10. Cardoso NG, Lopes LGG, Moreno LO, Muceniecks AGS. Aspectos psicológicos envolvidos na cirurgia cardíaca: uma revisão bibliográfica. Revista Científica Ágape 2018; 1(1): 1-6.

11. Nemer Bergmann AR, Vilela e Souza L, Scorsolini-Comin F, Santos MA. A vida por um fio: percepções sobre o implante de marca-passo cardíaco permanente. Revista Subjetividades 2016; 16(1): 131-143.

12. Angerami-Camon VA, organizador. Psicologia hospitalar: teoria e prática. São Paulo: Cengage Learning; 2009.

13. Bezerra SMMS, Gomes ET, Oliveira RC. Ser-paciente-à-espera-da-cirurgia-cardíaca: o período préoperatório na perspectiva heideggeriana. Rev Bras Enferm 2018; 71 (5): 2535-40. Disponível em: http://www.scielo.br/pdf/reben/v71n5/pt_0034-7167-reben-71-05-2392.pdf. Acesso em março de 2020.

14. Freud S. Reflexões para os tempos de guerra e morte. Rio de Janeiro: Imago; 1915.

15. Cintra MEU, Vieira MRJ. O trabalho criativo: perda, luto e metáfora. Gerais: Revista Interinstitucional de Psicologia 2016; 9(1): 50-66.

16. Grisa GH, Monteiro JK. Aspectos emocionais do paciente cardíaco cirúrgico no período préoperatório. Revista Interinstitucional de Psicologia 2015; 8(1): 111-130.

17. Luchina IL. La angustia de muerte em el infarto del miocárdio: sus implicaciones clinicas, psicologicas y psiquitricas. In: Blumenfield M, Tiamson-kassab M. Medicina Psicossomática. São Paulo: Artes Médicas; 1966. 